\title{
Influence of elastic 3D printed polymers on the mechanical properties and tribology of textile fabrics
}

\author{
Pia Steinmetz, Andrea Ehrmann* \\ Bielefeld University of Applied Sciences, Faculty of Engineering and Mathematics, Bielefeld, Germany \\ ${ }^{*}$ Corresponding author E-mail address: andrea.ehrmann@fh-bielefeld.de
}

\section{INFO}

CDAPT, ISSN 2701-939X

Peer reviewed article

2021, Vol. 2, No. 2, pp. 115-122

DOI 10.25367/cdatp.2021.2.p115-122

Received: 3 October 2021

Accepted: 6 November 2021

Available online: 6 November 2021

\begin{abstract}
Combining textile fabrics with $3 D$ printing has been investigated intensively during the last years. Mostly, research concentrated on the adhesion between both partners of the composite or on the new freedom of design, enabled by combining these techniques. Here, we present examinations of the influence of elastic $3 D$ printed patterns on the elongation and wearing out of elastic textile fabrics as well as on the tribological properties of the textile surface, comparing pure and imprinted textile fabrics. Therefore, thermoplastic polyurethane (TPU) was $3 D$ printed in different patterns on diverse textile fabrics. Our study shows that for a sufficient adhesion, reached by small enough nozzle-fabric distance, elastic 3D printed patterns can indeed improve the surface resistance against wear.
\end{abstract}

\author{
Keywords \\ 3D printing, \\ Thermoplastic polyurethane (TPU), \\ Woven fabric, \\ Knitted fabric, \\ Martindale test, \\ Abrasion
}

(C) 2021 The authors. Published by CDAPT.

This is an open access article under the CC BY-NC-ND license https://creativecommons.org/licenses/ peer-review under responsibility of the scientific committee of the CDAPT.

(C) 2021 CDAPT. All rights reserved.

\section{Introduction}

While 3D printing - also called additive manufacturing - belongs to the emerging technologies of our time and enables creating objects with new shapes at small numbers, the recent technologies still suffer from the problems that they are usually relatively slow, and that the mechanical properties are often reduced as compared to injection molded parts [1]. In the fused deposition modeling (FDM) process which is most often used in low-cost 3D printers, this problem is partly related to air voids between the strands of molten filament from which an object is formed [2].

Besides embedding fibers or filaments into the 3D printing polymer, there are some other attempts to improve the mechanical properties of FDM printed objects $[3,4]$. By combining FDM printing with textile fabrics, not only the tensile properties are improved, but it is also possible to prepare large areas in which 
only defined parts are modified by 3D printing, in this way producing larger fabrics with position-dependent tailored mechanical properties in a fast way.

An important prerequisite for preparing such composites is, as generally in composites, the fiber-matrix adhesion. This parameter is sensitive to diverse material and printing parameters, such as the textile surface structure which, together with the viscosity of the molten 3D printing polymer, defines the possible penetration of the polymer into the pores of the textile fabric [5-7]. The penetration depth is also influenced by the nozzle temperature and partly by the printing bed temperature [8]. A much higher impact, however, is given by the distance between nozzle and fabric, defining how strongly the polymer is mechanically pressed into the fabric $[9,10]$.

Thinking about a purely form-locking connection between both materials, using the low-viscosity resins applied for stereolithography (SLA) instead of the molten polymers used in FDM printing seems likely. Indeed, combining SLA with textile fabrics has been proven to be possible [11]. However, FDM printing has the advantages of mostly avoiding toxic resins and unhealthy evaporations as well as using less expensive material, making it easier usable in textile companies and for many of the available materials more environmentally-friendly.

Besides the form-locking connection, it is also possible to develop a chemical connection between both partners. For this, the fabrics can be pre-treated chemically or post-treated thermally [12-14]. On the other hand, the adhesion between elastic filaments, such as thermoplastic polyurethane (TPU), and textiles fabrics is often high enough to withstand washing and abrasion without further improvements [15-17].

Here, we used TPU printed on diverse textile fabrics to investigate the resistance of the polymer against abrasion using a Martindale abrasion tester. We tested the adhesion of the imprinted patterns and compared the surface morphology of the abraded textile fabrics with and without additional 3D printed material. For the stretchable textile fabrics, we also performed cyclic tensile tests to investigate whether the additional 3D prints lead to reduced residual stretch in comparison with pure textile fabrics, as shown for other patterns in a previous study [18].

\section{Methods and materials}

3D printing was performed with a CR-10 V2 (Creality, Shenzhen, China) with nozzle diameter $0.4 \mathrm{~mm}$. The filament Filaflex 82A (Recreus, Elda, Spain) was chosen for printing. The extruder temperature was set to $230{ }^{\circ} \mathrm{C}$, while the printing bed temperature remained at room temperature, to avoid different temperatures at the surfaces of textile fabrics with different thickness. A layer thickness of $0.1 \mathrm{~mm}$ was chosen to print the patterns depicted in Fig. 1 with height and width $1.5 \mathrm{~mm}$, respectively. All patterns were constructed without linear parts so that they could be extended on the elastic fabrics even without stretching of the polymer.

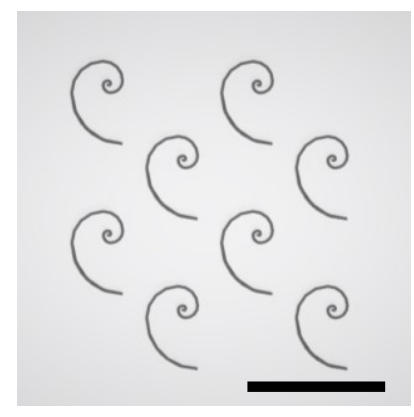

a

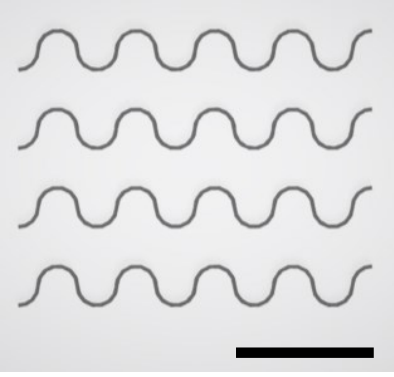

b

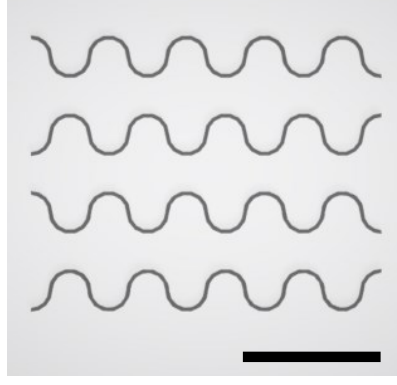

C

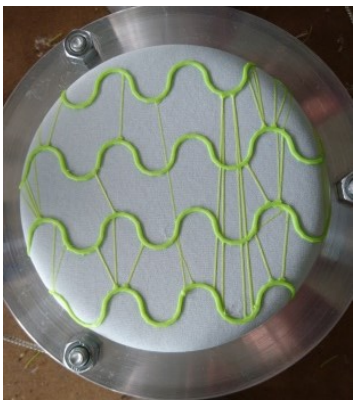

d

Fig. 1 (a) Fibonacci spiral; (b) parallel waves; (c) opposite waves; d) photograph of parallel waves on a soft-shell fabric (cf. Table 1). Scale bars show $50 \mathrm{~mm}$.

Since some of the examined fabrics were elastic, the following procedure was used to mount them on the printing bed: A cardboard was cut to the size of the printing bed. Parcel tape was used to fix the fabric edges on this cardboard, which was afterwards fixed on the printing bed by foldback clips. The elastic 
fabrics were slightly stretched so that they could still be fixed by the parcel tape, but were not drawn away from the nozzle during printing.

As mentioned before, one of the most important parameters defining the adhesion is the z-distance between nozzle and textile fabric. For each combination of filament and textile fabric, there is an optimum z-distance, as shown in previous studies $[9,10,15]$. Here, most samples were printed with optimum zdistance; however, in a few cases also slightly larger distances were investigated to show the influence of distance differences of only $0.1 \mathrm{~mm}$ or even less. The optimum z-distance was found by decreasing the zdistance slowly until the point where the polymer flow out of the nozzle stopped and then carefully increasing it again to the lowest nozzle height which allowed the printing material flowing out of the nozzle without disruption. This optimum value does not only depend on the fabric thickness, but also on its surface structure and the mechanical behavior of the fabric; for soft fabrics, printing "below the surface" of the textile is often possible, while dense fabrics often need larger distances. This is why the optimum zdistances used in this study differ between different fabrics.

The fabrics used in this study (purchased from fabfab $\mathrm{GmbH}$, Schenefeld, Germany) are depicted in Table 1. According to the fabric specifications, no additional surface coatings were applied which might influence the adhesion of 3D printed polymers. The jersey fabric can be expected to show much higher stretchability than the woven fabrics under examination.

Table 1. Textile fabrics used in this study. Horizontal side lengths of the micrographs are $1.55 \mathrm{~cm}$.

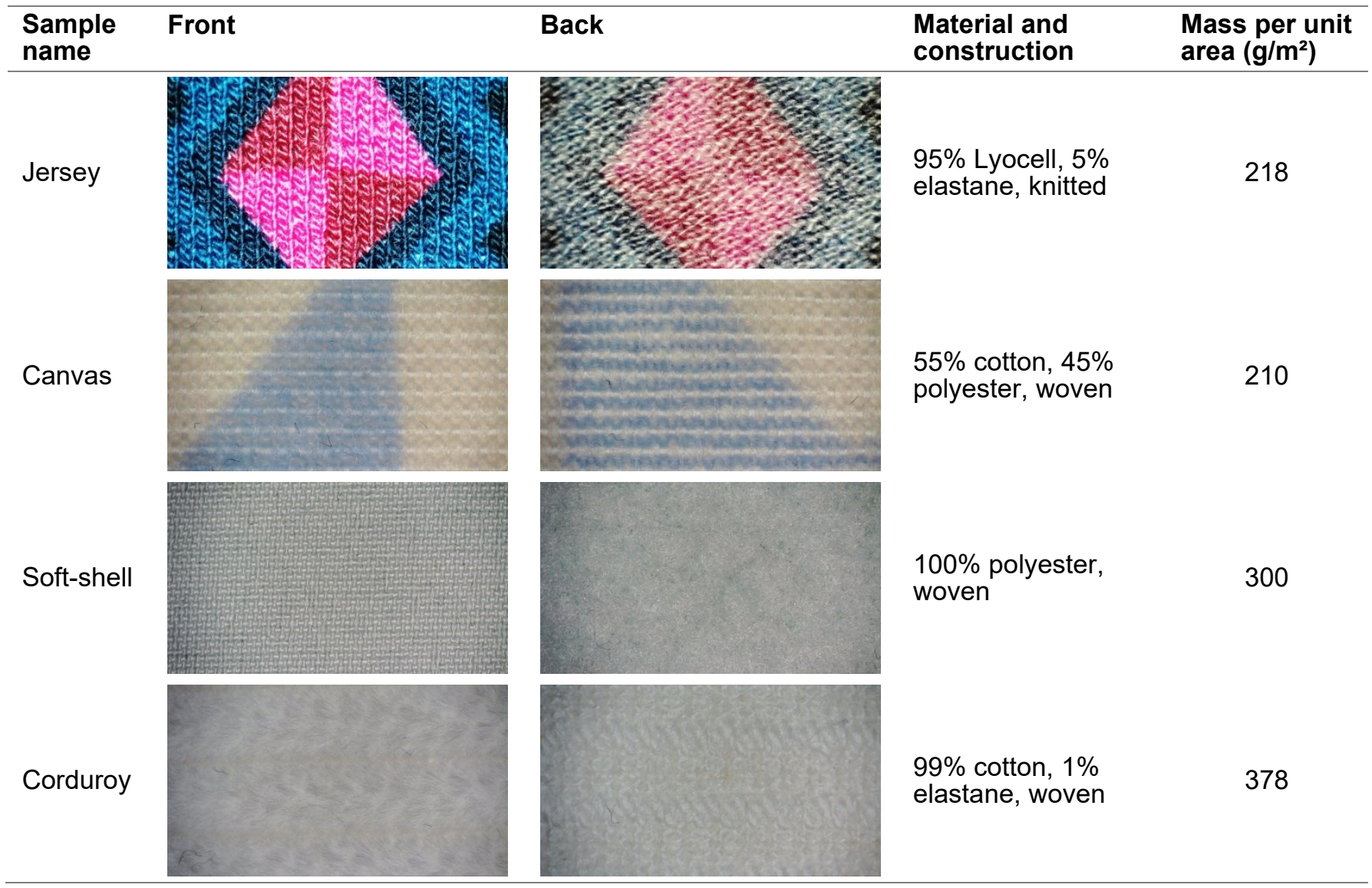

Microscopic images were taken with a digital microscope Camcolms2 (Velleman, Gavere, Belgium). Elongation tests were performed with a universal testing machine (Kern \& Sohn $\mathrm{GmbH}$, BalingenFrommern, Germany), using a free length between the clamps of $80 \mathrm{~mm}$. Abrasion of the textile surfaces was examined by a Martindale abrasion tester according to ISO 12947. During these tests, the fabrics with 3D prints were positioned in the lower, larger fixture, while the standard abrading fabric was mounted in the small upper fixture. Abraded parts were cut out of the lines to avoid collisions of the abrasion head with the 3D printed lines sticking out of the plane. 


\section{Results and discussion}

A necessary prerequisite to allow the 3D printed patterns modifying abrasion or mechanical properties of textile fabrics is given by the adhesion between polymer and textile fabric. Therefore, Fig. 2 depicts the numbers of abraded half-waves of the wave patterns (cf. Fig. 1b and c) after defined numbers of Martindale abrasion cycles. Since the test results were partly quite different for nominally identical samples, the single results of each test are shown instead of averaged values for nominally identical samples.
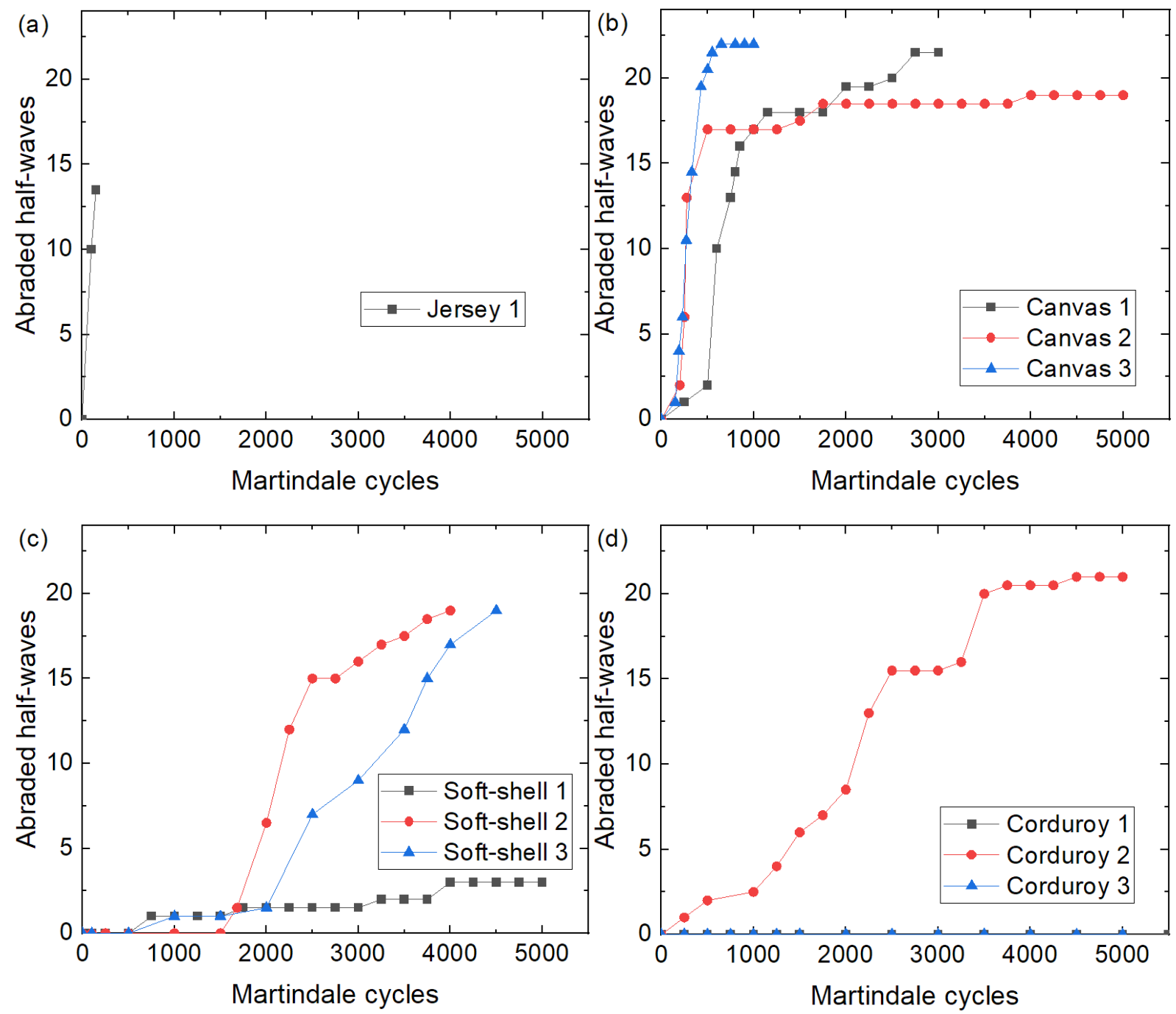

Fig. 2 Numbers of abraded 3D printed half-waves after up to 5000 Martindale cycles, measured on (a) a jersey fabric; (b) canvas fabrics; (c) soft-shell fabrics; and (d) corduroy fabrics.

For the highly elastic jersey fabric (Fig. 2a), this test was nearly impossible to perform since the textile was strongly moved by the abrading head as soon as a 3D printed line was touched. The test shown here was thus stopped after 150 Martindale cycles. This problem did not occur for the other, nearly inelastic fabrics.

On the canvas fabric (Fig. 2b), the imprinted polymer was relatively fast lost, with most of the 25 halfwaves reachable by the abrasion head being lost after few hundred up to few thousand Martindale cycles. The results show, however, that in some cases parts of the 3D printed patterns survive even after 5000 Martindale cycles. These residual patterns can generally be found along the outer parts of the patterns, i.e. in areas which are only partly touched by the abrading head and which are additionally fixed by the parts of the lines which are outside the movement area of the abrading head.

For the soft-shell (Fig. 2c) and especially the corduroy samples (Fig. 2d), different results are visible. Here, depending on the z-distance, the adhesion is quite good or even perfect during 5000 Martindale cycles; for corduroy, tests up to 10,000 cycles showed the same result. In both cases, the differences between 
perfect z-distance (black line in Fig. 2c, black and blue lines in Fig. 2d) and a z-distance increased by approx. $0.1 \mathrm{~mm}$ is clearly visible. The importance of this parameter cannot be overemphasized, making it necessary to find the perfect distance for each new fabric experimentally instead of just guessing the best setting.

Besides this basic investigation of the adhesion, one of the aims of this study was the evaluation of the suitability of elastic 3D prints on textile fabrics to protect the surfaces from abrasion damages. Fig. 3 shows images of the surfaces after abrasion tests with and without protection by 3D printed lines.

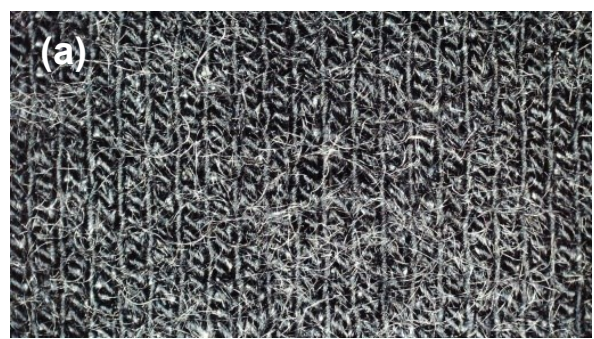

(d)

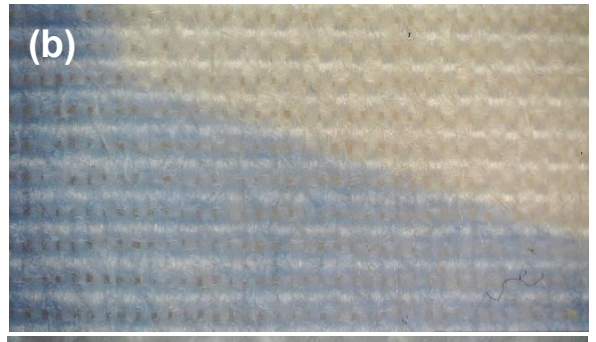

(e)

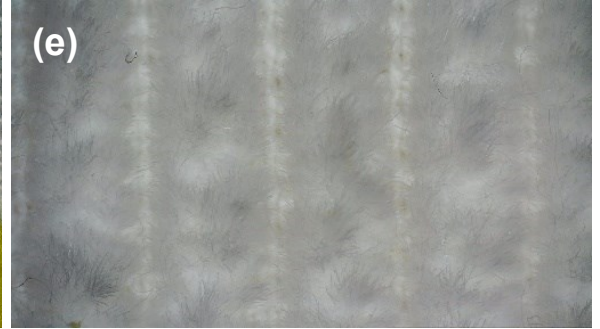

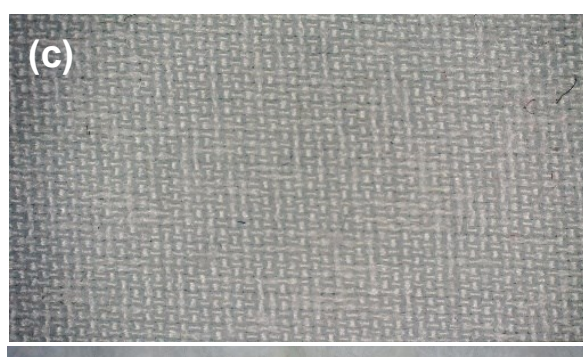

(f)

Fig. 3 Microscopic images of different fabrics after Martindale tests: (a) jersey fabric after 150 Martindale cycles without 3D printed lines; (b) canvas fabric after 5000 Martindale cycles without 3D printed lines; (c) soft-shell fabric after 5000 Martindale cycles without 3D printed lines; (d) soft-shell fabric after 5000 Martindale cycles with 3D printed lines; (e) corduroy fabric after 5000 Martindale cycles without 3D printed lines; and (f) (e) corduroy fabric after 10,000 Martindale cycles with $3 D$ printed lines.

For the jersey fabric, even after only 150 Martindale cycles the first pilling is visible (Fig. 3a), with a large number of hairs protruding out of the fabric surface. As described above, however, testing the influence of a 3D printed pattern on the abrasion of this surface was not possible since the fabric was too elastic for the Martindale test.

The canvas fabric showed nearly no surface modification after 5000 Martindale cycles without 3D printed lines (Fig. 3b); thus testing the influence of a 3D printed pattern would necessitate longer tests which the 3D prints could not withstand (cf. Fig. 2b).

Similarly, on the soft-shell fabric there is no impact on the surface visible after 5000 Martindale cycles (Fig. 3c). The 3D printed lines are also mostly unchanged (Fig. 3d).

Only on the corduroy fabric, the surface looks "fluffier" after 5000 Martindale cycles, and can be estimated from Fig. 3e, which can indeed be avoided near the 3D printed lines even after 10,000 cycles (Fig. 3f).

As these results shown, using 3D printed patterns for abrasion protection of textile surfaces is not easy. On the one hand, it is necessary to combine fabric and polymer in such a way that the adhesion between both parts is large enough; on the other hand, additional 3D printing can even enlarge damage upon abrasion if the abrading object sticks to the 3D printed patterns and tears at them much more, than it would be possible on the pure textile surface. Apparently, this approach needs to be tested carefully for each textile fabric. It should be mentioned that these results were found similar for the Fibonacci spiral (Fig. 1a) which was not further investigated since quantifying the abraded parts of a pattern was more problematic than for the wave-like patterns.

Next, tensile tests were performed with the two elastic fabrics, i.e. jersey (bi-elastic) and soft-shell (slightly elastic in one direction). Fig. 4a depicts tensile tests until breaking of the soft-shell fabric with and without additional 3D printed waves parallel to the tension direction. In all tests $(n=3)$, the fabrics showed very 
similar forces, where the maximum force was stronger influenced by the cutting direction (exactly along the woven threads or under a small angle, which could happen since the threads are not well visible on a macroscopic scale) than by the 3D printed lines. For the exemplary measurements depicted in Fig. 4a (the average results of each set of measurements), the force at break is even slightly smaller for the fabric with addition 3D print than for the pure fabric, while the elongation at break is approximately identical.
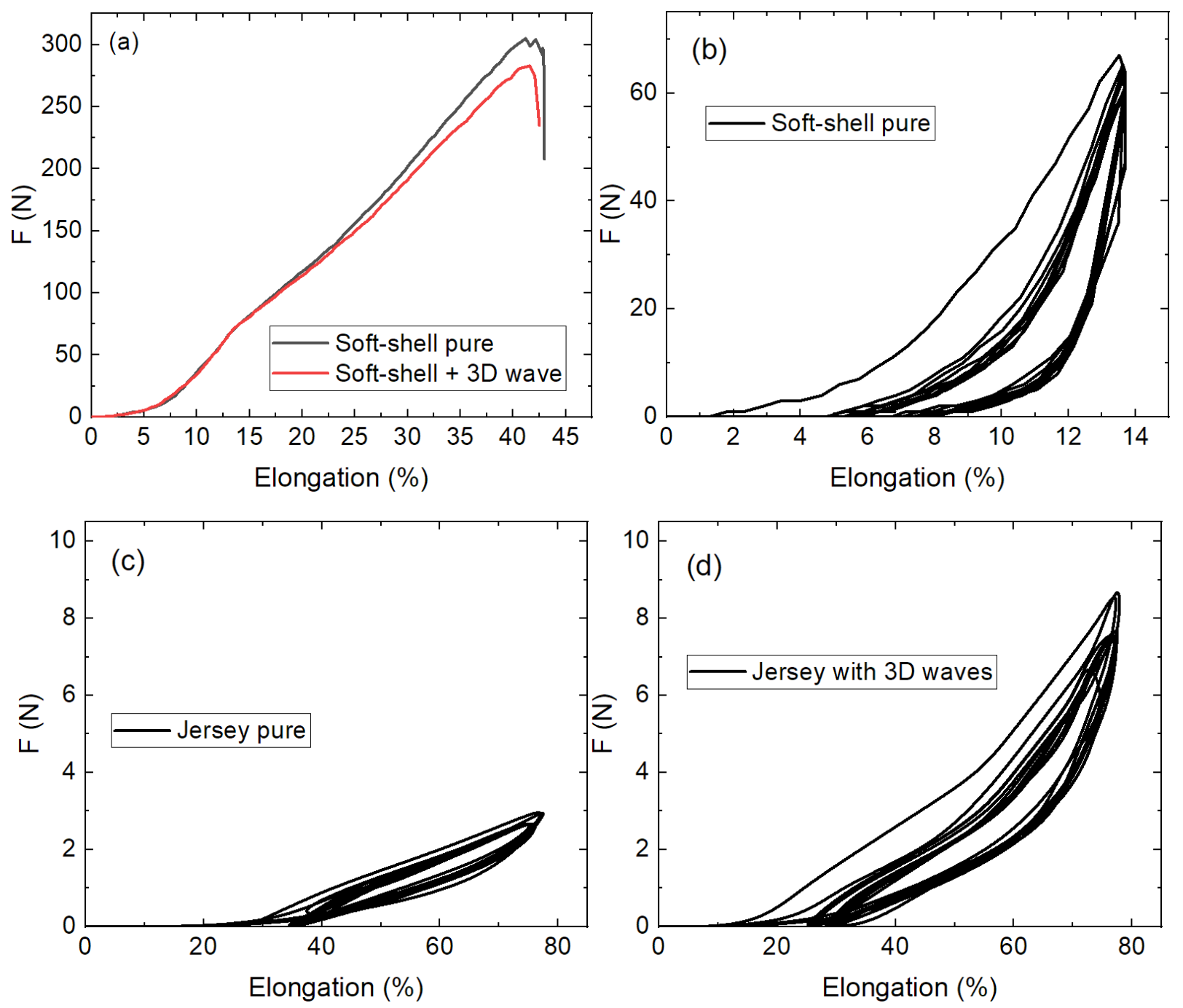

Fig. 4 (a) Tensile tests on pure soft-shell fabric and soft-shell with 3D printed lines; cyclic tensile tests (10 cycles each) on (b) a pure soft-shell fabric; (c) a pure jersey fabric; and (d) a jersey fabric with $3 D$ printed waves.

Due to the change of the slope near $14 \%$ elongation (Fig. 4a), the cyclic tensile tests of the soft-shell fabrics were reversed at this elongation. Fig. $4 \mathrm{~b}$ shows the results of 10 cyclic tests, with a clearly different first cycle with larger force and a residual elongation of approximately $5 \%$, which does not change strongly during the next nine cycles. The measurement on a soft-shell fabric with additional 3D printed line parallel to the tension direction shows nearly the same result (not shown here). In case of this slightly elastic fabric, the additional imprinted polymer lines thus do not change the tensile behavior of the samples, neither in cyclic tests nor in tests till the fabric breaks.

Both jersey fabrics with and without additional 3D printed lines were highly elastic and did not break within the maximum tension, defined by the dimensions of the test instrument, so that no test of the maximum force at break are shown here. Here, opposite to the soft-shell fabric, a clear influence of an additional 3D printed pattern parallel to the tension direction is visible in Figs. 4c and 4d. The maximum force is nearly tripled by the 3D printed pattern. While Fig. 4d depicts a cyclic measurement of two parallel waves (Fig. $1 \mathrm{~b}$ ), the results of cyclic measurements with two antiparallel waves (Fig. 1c) are identical within the measurement accuracy. The Fibonacci spirals were found to cause irreproducible deformations of the 
textile fabrics and were thus not further tested. Apparently 3D printed patterns are able to influence the mechanical properties of highly elastic fabrics.

It must be mentioned that while abrasion tests on the jersey fabrics with 3D printed patterns were nearly impossible, the cyclic tensile tests - which stretched the waves nearly to perfect lines - were unproblematic and did not detach the 3D printed waves from the fabrics. This shows that the optimum test method has to be chosen according to the planned application.

\section{Conclusions and outlook}

In a recent study, TPU patterns were 3D printed on different textile fabrics. Abrasion and tensile tests showed that the TPU adhered with different strength, depending on the fabric and especially on smallest variations of the z-distance between nozzle and fabric.

While the most elastic jersey fabric could not be tested properly by a Martindale abrasion tester, and two of the woven fabrics did not show surface modifications after the test without additional 3D printed pattern, for the fourth fabric a clear difference was visible after abrasion tests on the pure surface and on the fabric with imprinted 3D lines, showing that in principle 3D printed patterns can be used to protect textile surfaces from undesired modifications upon abrasion, as long as the adhesion between both materials is sufficient.

Tensile tests with a slightly elastic woven fabric and the highly elastic jersey showed that the mechanical properties of the latter were significantly influenced by adding 3D printed lines.

These examinations show that combinations of 3D printed TPU patterns may influence the textile fabrics in terms of mechanical properties or protect the surface from undesired effects due to abrasion. In any case, however, optimizing the z-distance to reach sufficient adhesion between both materials is a necessary prerequisite. The importance of this printing parameter suggests more research on it to enable optimizing this value without performing a test series in advance, e.g. by measuring the force of the textile fabric counteracting the nozzle or the polymer flow.

\section{References}

[1] Oviedo, A. M.; Puente, A.H.; Bernal, C.; Perez, E. Mechanical evaluation of polymeric filaments and their corresponding 3D printed samples. Polymer Testing 2020, 88, $106561 . \quad$ DOI 10.1016/j.polymertesting.2020.106561.

[2] Meyer, P.; Döpke, C.; Ehrmann, A. Improving adhesion of 3D printed objects on textile fabrics by polymer coating. J. Eng. Fibers Fabr. 2019, 14, 1558925019895257. DOI 10.1177/1558925019895257.

[3] Arunothayan, A. R.; Nematollahi, B.; Ranade, R.; Bong, S. H.; Sanjayan, J. Development of 3D-printable ultrahigh performance fiber-reinforced concrete for digital construction. Construction and Building Materials 2020, 257, 119546. DOI 10.1016/j.conbuildmat.2020.119546.

[4] Afshar, A.; Mihut, D. Enhancing durability of 3D printed polymer structures by metallization. J. Mater. Sci. Technol. 2020, 53, 185-191. DOI 10.1016/j.jmst.2020.01.072.

[5] Narula, A.; Pastore, C.; Schmelzeisen, D.; El Basri, S.; Schenk, J.; Shajoo, S. Effect of knit and print parameters on peel strength of hybrid 3D printed textiles. J. Text. Fibrous Mater. 2018, 1: 2515221117749251. DOI $10.1177 / 2515221117749251$.

[6] Calvo, J.O.; Martin, A.C.; Ferradas, M.I.R.; Morcillo, P.L.F.; Munoz, L.M.; Camo, P.M. Additive manufacturing on textiles with low-cost extrusion devices: Adhesion and deformation properties. Dyna 2019, 64, 8893. DOI 10.6036/8893.

[7] Mpofu, N. S.; Mwasiagi, J. I.; Nkiwane, L. C.; Njuguna, D. Use of regression to study the effect of fabric parameters on the adhesion of 3D printed PLA polymer onto woven fabrics. Fashion and Textiles 2019, 6, 24. DOI 10.1186/s40691-019-0180-6.

[8] Eutionnat-Diffo, P. A.; Chen, Y.; Guan, J. P.; Cayla, A.; Campagne, C.; Zeng, X. Y.; Nierstraz, V. Stress, strain and deformation of poly-lactic acid filament deposited onto polyethylene terephthalate woven fabric through 3D printing process. Sci. Rep. 2019, 9, 14333. DOI 10.1038/s41598-019-50832-7.

[9] Grimmelsmann, N.; Kreuziger, M.; Korger, M.; Meissner, H.; Ehrmann, A. Adhesion of 3D printed material on textile substrates. Rapid Prototyping J. 2018, 24(1), 166-170. DOI 10.1108/RPJ-05-2016-0086.

[10] Spahiu, T.; Al-Arabiyat, M.; Martens, Y.; Ehrmann, A.; Piperi, E.; Shehi, E. Adhesion of 3D printing polymers on textile fabrics for garment production. IOP Conf. Ser.: Mater. Sci. Eng. 2018, 459, 012065. DOI: 10.1088/1757$899 \times / 459 / 1 / 012065$. 
[11] Grothe, T.; Brockhagen, B.; Storck, J. L. Three-dimensional printing resin on different textile substrates using stereolithography: A proof of concept. J. Eng. Fibers Fabrics 2020, 1, 1558925020933440. DOI: $10.1177 / 1558925020933440$.

[12] Korger, M.; Bergschneider, J.; Lutz, M.; Mahltig, B.; Finsterbusch, K.; Rabe, M. Possible Applications of 3D Printing Technology on Textile Substrates. IOP Conf. Ser.: Mater. Sci. Eng. 2016, 141, 012011. DOI 10.1088/1757-899X/141/1/012011.

[13] Kozior, T.; Döpke, C.; Grimmelsmann, N.; Juhász Junger, I.; Ehrmann, A. Influence of fabric pretreatment on adhesion of three-dimensional printed material on textile substrates. Adv. Mech. Eng. 2018, 10, 792316. DOI $10.1177 / 1687814018792316$.

[14] Störmer, J.; Görmer, D.; Ehrmann, A. Influence of washing and thermal post-treatment on the adhesion between 3D-printed TPU and woven fabrics. CDATP 2021, 2, 34-39. DOI 10.25367/cdatp.2021.2.p34-39.

[15] Görmer, D.; Störmer, J.; Ehrmann, A. The influence of thermal after-treatment on the adhesion of 3D prints on textile fabrics. Communications in Development and Assembling of Textile Products 2020, 1, 104-110.

[16] Korger, M.; Glogowsky, A.; Sanduloff, S.; Steinem, C.; Huysman, S.; Horn, B.; Ernst, M.; Rabe, M. Testing thermoplastic elastomers selected as flexible three-dimensional printing materials for functional garment and technical textile applications. J. Eng. Fibers Fabrics 2020, 15, $1558925020924599 . \quad$ DOI $10.1177 / 1558925020924599$.

[17] Zedler, S. L. Structural compressibility in 3D printed abrasion protection structures. Communications in Development and Assembling of Textile Products 2020, 1, 3-11. DOI 1 0.25367/cdatp.2020.1.p3-11.

[18] Ayvali, M.; Bussieweke, L.; Druzinin, G.; Korkmaz, M.; Ehrmann, A. 3D printing on warp-knitted fabrics. IOP Conf. Series Mater. Sci. Eng. 2021, 1031, 012019. DOI 10.1088/1757-899X/1031/1/012019. 